\title{
Effect of the labelling ratio on the photophysics of fluorescein isothiocyanate (FITC) conjugated to bovine serum albumin $\uparrow$
}

\author{
Graham Hungerford, ${ }^{* a}$ Johan Benesch, ${ }^{b}$ João F. Mano ${ }^{b, c}$ and Rui L. Reis ${ }^{b, c}$ \\ Received 6th September 2006, Accepted 15th November 2006 \\ First published as an Advance Article on the web 20th December 2006 \\ DOI: 10.1039/b612870j
}

The non-linearity of the fluorescence emission on increasing the probe to protein ratio has long been regarded as problematic and has lead to the development of dyes to overcome this effect. One of the causes of this non-linear response can be ascribed to the overlap of the label's own absorption and emission spectra. At higher labelling ratios, this affords the possibility of a reasonably efficient energy migration pathway, thus reducing the observed quantum yield of the dye. In this work we study the photophysics of fluorescein isothiocyanate (FITC) when conjugated to bovine serum albumin (BSA) at different labelling ratios (in the range FITC:BSA $1: 17-15: 1$ ) using both steady state and time-resolved fluorescence techniques where on going from under labelled to over labelled samples a decrease in the initial (and steady state) anisotropy is observed, accompanied by an increase in the complexity of the decay kinetics and a decrease in the average lifetime. The band structure, elucidated by synchronous scan fluorescence spectroscopy, is also found to change on increased labelling. These results can be applied to the study of protein conformation and were confirmed by the analysis of denaturing BSA using urea.

\section{Introduction}

The observation of a reduction in quantum yield on increasing the amount of a fluorescent label on a protein has been often thought of as problematic. However, one of the underlying causes, that of energy migration, can in fact be turned to an advantage in the study of protein conformation. Fluorescence resonance energy transfer has long been employed as a nanoscale molecular ruler. ${ }^{1}$ This phenomenon usually makes use of donor and acceptor molecules chosen such that the donor emission spectrum overlaps with the absorption spectrum of the acceptor to facilitate energy transfer via the Förster mechanism. ${ }^{2}$ To label a protein with individual donor and acceptor moieties in itself is not straightforward in comparison to tagging with a single fluorescent label. However, mixing singly tagged bovine serum albumin (BSA) molecules, one with a donor and the other with an acceptor has been used to observe protein dimerization. ${ }^{3}$ The technique of using energy migration between like fluorophores has been successfully applied to measure distances proteins, ${ }^{4-6}$ in lipid bilayers ${ }^{7}$ and to study protein unfolding. ${ }^{8}$

Fluorescein isothiocyanate (FITC) is a fluorescein derivative, which is commonly used as an amine reactive fluorescence label. The photophysics of fluorescein is known to be $\mathrm{pH}$ dependent, ${ }^{9}$ and it exhibits a reasonable overlap between its absorption and

${ }^{a}$ Departamento de Fisica, University of Minho, Campus de Gualtar, 4710057, Braga, Portugal. E-mail: graham@fisica.uminho.pt; Fax: 00351253678981; Tel: 00351-253604320

${ }^{b}$ The 3 Bs Research Group - Biomaterials, Biodegradables and Biomimetics, Department of Polymer Engineering, University of Minho, Campus de Gualtar, 4710-057, Braga, Portugal

'Department of Polymer Engineering, University of Minho, Campus de Azurém, 4800-058, Guimarães, Portugal

$\dagger$ Electronic supplementary information (ESI) available: Absorption spectra of FITC-BSA at different labelling ratios. See DOI: 10.1039/b612870j emission spectra and a critical energy transfer distance ${ }^{1}$ in the range 42 to $56 \AA$ have been reported. ${ }^{10-12}$ This makes it a suitable candidate to study protein conformation (in non-acidic media) by making use of donor-donor energy migration. Although this phenomenon of "self quenching" is widely known, there is a surprising lack of published detailed reports relating to it, especially as FITC is a commonly used probe.

In this work we have studied the photophysics of FITC when conjugated to varying degrees to BSA. The time-resolved kinetics was studied using single-photon counting, ${ }^{13}$ and the effect of energy migration on both the steady state and time-resolved anisotropy was monitored. Further information concerning intermolecular interactions was ascertained using synchronous scan fluorescence spectroscopy (SFS). ${ }^{14}$

\section{Experimental}

\section{Dye-protein conjugation}

Bovine serum albumin, fraction V, 96\% (Sigma) was labelled with fluorescein isothiocyanate (Sigma) in $0.1 \mathrm{M}$ carbonate buffer $(\mathrm{pH} 9)$ at room temperature and in the dark for $4 \mathrm{~h}$ and with a labelling ratio $(\mathrm{F}: \mathrm{P})$ in the range $1: 10$ to $10: 1$. The FITCBSA solutions were dialysed with cellulose membranes (Sigma, cut-off $12 \mathrm{kDa}$ ) against $1 \mathrm{~L}$ of $0.01 \mathrm{M}$ phosphate buffered saline (PBS) at $\mathrm{pH} 7.4$ four times for $1 \mathrm{~d}$ each in the dark at $8^{\circ} \mathrm{C}$ to remove any uncoupled FITC. The light absorption at $495 \mathrm{~nm}$ was finally below 0.003 for the supernatant. The dialysis membranes were washed prior to use in distilled water for at least $4 \mathrm{~h}$ with extensive rinsing once every hour. The concentration and $\mathrm{F}: \mathrm{P}$ ratio were determined according to the methods described by the manufacturer using the albumin extinction coefficient at $280 \mathrm{~nm}$, $0.66 \mathrm{mg}^{-1} \mathrm{ml}^{-1}$ and a ratio of FITC absorption at 280 versus $495 \mathrm{~nm}$ of 0.35 . Samples were diluted in such a way to keep the amount of 
Table 1 Peak absorption and emission of FITC when coupled at different ratios to BSA

\begin{tabular}{|c|c|c|c|c|c|c|}
\hline FITC: BSA & FITC/BSA & Absorption $\lambda_{\max } / \mathrm{nm}$ & $\mathrm{abs}_{502} / \mathrm{abs}_{477}$ & Emission $\lambda_{\max } / \mathrm{nm}$ & $I_{518} / I_{541}$ & Stokes' shift/nm \\
\hline $1: 17$ & 0.058 & 502 & 2.8 & 518 & 2.2 & 16 \\
\hline $1: 10$ & 0.1 & 502 & 2.3 & 516 & 2.4 & 14 \\
\hline $1: 4$ & 0.26 & 502 & 2.9 & 519 & 2.0 & 15 \\
\hline $1: 2$ & 0.47 & 502 & 2.9 & 516 & 2.3 & 14 \\
\hline $1: 1 \mathrm{a}$ & 0.8 & 502 & 2.7 & 518 & 2.2 & 16 \\
\hline $1: 1 b$ & 0.97 & 502 & 2.9 & 518 & 2.2 & 16 \\
\hline $2: 1 \mathrm{a}$ & 1.81 & 503 & 2.8 & 518 & 2.1 & 15 \\
\hline $2: 1 b$ & 1.97 & 503 & 2.8 & 519 & 2.1 & 16 \\
\hline $3: 1 \mathrm{a}$ & 2.54 & 502 & 2.5 & 520 & 1.9 & 18 \\
\hline $3: 1 b$ & 2.78 & 502 & 2.3 & 520 & 1.8 & 18 \\
\hline $3: 1 \mathrm{c}$ & 2.8 & 499 & 1.7 & 521 & 1.7 & 22 \\
\hline $7: 1$ & 7.2 & 499 & 1.8 & 521 & 1.6 & 22 \\
\hline $10: 1$ & 10.2 & 500 & 2.1 & 522 & 1.5 & 22 \\
\hline $15: 1$ & 14.9 & 498 & 1.6 & 523 & 1.4 & 25 \\
\hline
\end{tabular}

FITC constant (absorbance $c a$. 0.2 ) as the principle purpose is to study its photophysics, hence the under labelled samples contain a higher concentration of protein than the over labelled ones. Inverse micelles were made using a stock solution of $0.25 \mathrm{M}$ sulfosuccinic acid bis(2-ethylhexyl) ester, sodium salt (AOT), from Sigma, in trimethyl pentane (TMP), from Aldrich. FITC was then added in PBS (to obtain the required surfactant: water ratio, $\omega_{0}$, value). Urea was purchased from Sigma and used as received.

\section{Measurements and analysis}

Spectra were recorded using a Shimadzu UV-3101PC (absorption) and a SPEX Fluorolog II (fluorescence and synchronous scan). Measurements were made at room temperature. The spectral analysis of the steady state emission spectra was carried out using Microcal Origin 7 software to perform the decomposition of the measured spectra as a sum of Gaussian components, in agreement with previous studies. ${ }^{15,16}$ Where required, correction was made for any inner filter effects. ${ }^{17}$

Fluorescence lifetimes were measured by means of a singlephoton counting apparatus equipped with a LED excitation source with a peak emission at $495 \mathrm{~nm}$ (HORIBA, Jobin Yvon, IBH Ltd., Glasgow, Scotland). The fluorescence emission was wavelength selected using a $550 \mathrm{~nm}$ cut-off filter and detected with a Hamamatsu R2949 photomultiplier. Data were analysed as the sum of exponential components (eqn (1)), with the preexponential components $\left(a_{i}\right)$ normalised to unity, performed using IBH DAS6 software. Errors are taken as three standard deviations and the goodness of fit judged in terms of a $\chi^{2}$ value and weighted residuals.

$$
I(t)=\sum_{i=1}^{n} \alpha_{i} \exp \left(-t / \tau_{i}\right)
$$

\section{Results and discussion}

\section{Steady state absorption and fluorescence}

On coupling to the protein a bathochromic shift in the peak of the FITC absorption is observed (from $493 \mathrm{~nm}$ to around $502 \mathrm{~nm}$ ). This type of behaviour has previously been reported for both absorption and emission spectra and is related to the effect of the local environment. ${ }^{18,19}$ This shift, relative to the free fluorescein derivative, remains consistent for the under labelled, however, when the labelling ratio is close to $3: 1$ (FITC:BSA) a decrease in the peak absorption wavelength is observed along with an increase in its full width at half maximum (FWHM), see Table 1 and Fig. 1. It is worthy to note that there is also a relative increase in the shoulder located at shorter wavelengths to the peak absorption with increasing labelling ratio.

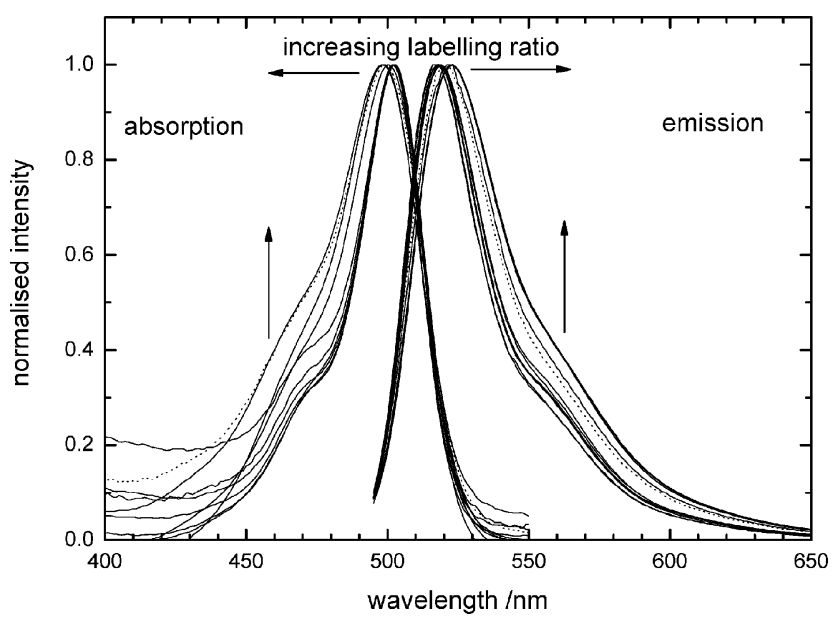

Fig. 1 Normalised absorption and emission spectra for FITC-BSA (at FITC: BSA, 1:17, 1:10, 1:4, 1:2, 1:1b, 2:1b, 3:1c, $7: 1,10: 1,15: 1$ ).

The fluorescence emission (Table 1 and Fig. 1) also exhibits a trend in the over labelled samples. In this case there is also an increase in the FWHM of the emission, but the shift is bathochromic, which results in an apparent increase in the Stokes' shift. It is interesting to note that the shorter wavelength regions of the spectra are practically coincident and that the increase in width occurs at the longer wavelength side and that the shoulder in this region increases with increasing labelling ratio. A possible explanation is the increased interaction between the fluorophores. A broadening and an increase in the shoulder in the fluorescein absorption spectrum have previously been related to aggregation, ${ }^{20,21}$ and accompanied by a similar trend in the emission. $^{22}$

Decomposition of the spectra into a sum of Gaussian component spectra revealed two major bands, one centred at $502 \mathrm{~nm}$ and the other at $477 \mathrm{~nm}$ in the case of the absorption and in the case 
of the fluorescence spectra bands centred at $518 \mathrm{~nm}$ and $541 \mathrm{~nm}$ were found. Relative increases in the intensities of the $477 \mathrm{~nm}$ and $541 \mathrm{~nm}$ bands (evident in Fig. 1) were found with increased labelling. To quantify this, a simplistic approach of comparing the ratios of these wavelengths, in both the absorption and emission spectra was taken, see Table 1 . The drop of this ratio at $3: 1$ and above indicates a noticeable increase in the interaction between FITC molecules.

To further investigate the effect of concentration, a high concentration of uncoupled FITC $\left(\mathrm{ca} .5 \times 10^{-2} \mathrm{M}\right)$ was investigated in AOT inverse micelles $\left(\omega_{0}=5\right)$ and the resulting spectra are shown in Fig. 2. These clearly show that an increase in concentration leads to a now dominant band appearing on the shorter wavelength side of the absorption spectrum and a corresponding band on the longer wavelength side of the emission. Decomposition of the latter yielded two major bands at $515 \mathrm{~nm}$ and $545 \mathrm{~nm}$ for the fluorescence, which are comparable with those observed in the FITC-BSA system. Fitting the absorption spectrum was more complex and three main bands were recovered at $485 \mathrm{~nm}, 458 \mathrm{~nm}$ and $436 \mathrm{~nm}$. However, in light of these data it is clear that the behaviour observed in the steady state spectra with increased labelling results from the interaction between FITC molecules.

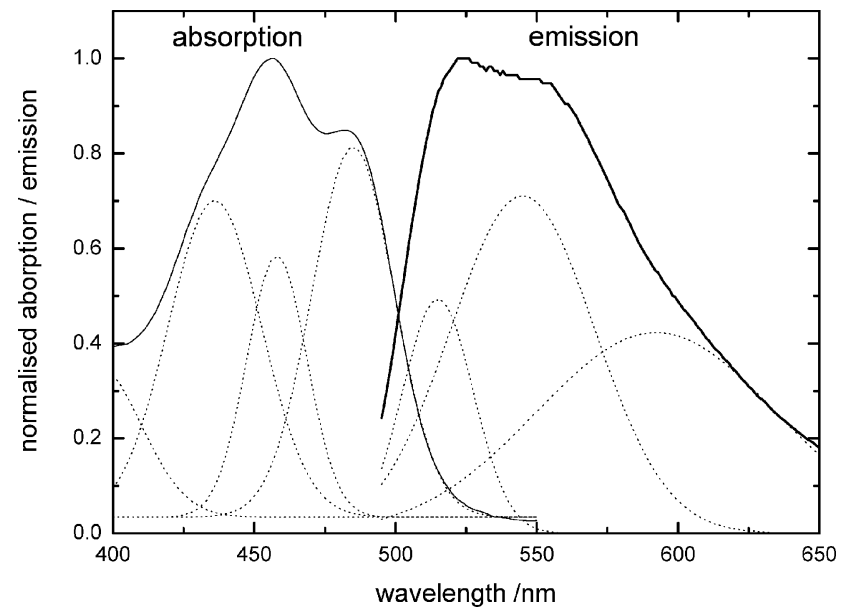

Fig. 2 Normalised absorption and emission spectra for uncoupled FITC in an inverse micelle system of PBS-AOT-TMP at $\omega_{0}=5$. The component spectra from the decomposition analysis are shown as dotted lines.

\section{Steady state fluorescence anisotropy}

Because the spectral overlap between the absorption and emission spectra of FITC is significant an increase in the possibility of an energy migration pathway between the FITC labels is expected when increasing the labelling ratio. This would be apparent as a decrease in anisotropy with increased labelling and Fig. 3 shows that this is, indeed, the case. At ratios below unity the steady state anisotropy is close to the maximum expected (0.4). We have divided the graph into three main regions (A, B and C). In the first region (A), where the samples are "under labelled" there is a relatively stable and high anisotropy value. The second region (B) on small increases in the labelling ratio, a significant decrease in the anisotropy in observed and finally in region $\mathrm{C}$ where there is "over labelling", further increases in the labelling ratio do not

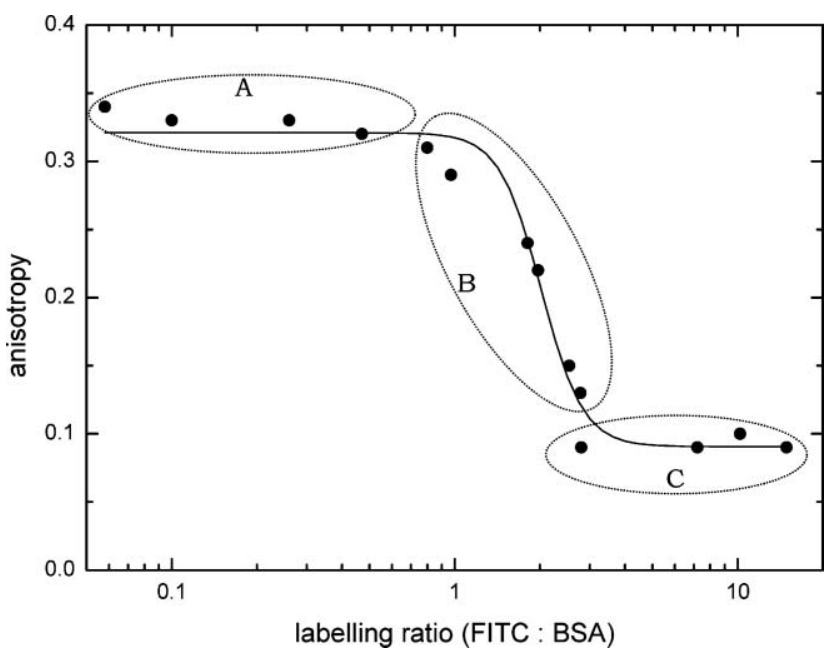

Fig. 3 Steady state anisotropy for FITC-BSA at different labelling ratios.

result in any noticeable change in the value of anisotropy. This type of behaviour is indicative of energy migration. ${ }^{5}$

\section{Synchronous scan fluorescence spectroscopy}

In order to further assess any possible inter-label interactions, such as the possibility of some dimer formation (as the Stokes' shift for monomer and dimer are different) a synchronous scan was performed on some selected samples, one from region A (see Fig. 3), with a FITC: BSA ratio of $1: 10$, three from region $B$ $(1: 1 \mathrm{~b}, 2: 1 \mathrm{~b}, 3: 1 \mathrm{~b})$ and one from region $\mathrm{C}(7: 1)$. First to establish the behaviour of uncoupled FITC a dilute solution was measured along with that concentrated within AOT inverse micelles and the outcome for different offsets between the excitation and emission wavelengths are given in Fig. 4. In this type of spectroscopy the observed intensity $\left(I_{\mathrm{s}}\right)$ is given by eqn. $(2)^{23}$

$$
I_{\mathrm{s}}=K c d E_{\mathrm{X}}(\lambda-\Delta \lambda) E_{\mathrm{M}}(\lambda)
$$

where $K$ is a constant, $c$ is the concentration, $d$ is the sample thickness, $E_{\mathrm{X}}(\lambda)$ is the intensity distribution of the excitation spectrum, $E_{\mathrm{M}}(\lambda)$ is the intensity distribution of the emission spectrum and the offset $\Delta \lambda$ has a constant value.

The first noticeable difference between the two samples is the relative differences in the intensities of the spectra at different values of offset. For example in the higher concentration sample the intensity of the $50 \mathrm{~nm}$ offset spectrum is equivalent to that of the $25 \mathrm{~nm}$ spectrum. In the lower concentration sample the equivalent spectral intensity is roughly half, which is expected because of the intensity of the shoulder attributed to dyedye interactions in the higher concentration sample. To extract additional information each of the spectra was decomposed into the sum of Gaussian component spectra and the outcome of this analysis is given in Table 2. Generally, the higher concentration sample required fewer Gaussian component spectra to adequately fit the spectra observed from the different offsets. At an offset of $25 \mathrm{~nm}$ (close to the Stokes' shift) both concentrations fit well to a single Gaussian spectrum. At increased wavelength offsets further components are required, with an extra one needed for the more dilute sample at offsets of $50 \mathrm{~nm}$ and $75 \mathrm{~nm}$ in relation to the higher concentration sample. 
Table 2 Fitting of the SFS spectra to the sum of Gaussian component spectra, showing the component peak positions, their contribution to the total intensity and the total intensity in comparison to that of the $25 \mathrm{~nm}$ offset spectrum $\left(I_{25 \mathrm{~nm}}\right)$ for different offsets between the excitation and emission wavelengths

\begin{tabular}{|c|c|c|c|c|c|c|c|c|c|c|}
\hline Labelling ratio $^{a}$ & Offset/nm & Peak 1/nm & Peak 2/nm & Peak 3/nm & Peak 4/nm & I $1(\%)$ & $I 2(\%)$ & I $3(\%)$ & I $4(\%)$ & $\%$ of $I_{25 \mathrm{~nm}}$ \\
\hline Free & 25 & 492 & & & & 100 & & & & \\
\hline FITC & 50 & 489 & 478 & 464 & & 63 & 5 & 32 & & 86 \\
\hline$[\mathrm{L}]$ & 100 & 487 & 459 & 430 & 407 & 36 & 41 & 18 & 5 & 23 \\
\hline Free & 25 & 489 & & & & 100 & & & & \\
\hline FITC & 50 & 486 & 461 & & & 59 & 41 & & & 167 \\
\hline$[\mathrm{H}]$ & 75 & 483 & 459 & 440 & & 45 & 23 & 32 & & 190 \\
\hline \multirow{3}{*}{$1: 10$} & 50 & 500 & 484 & 469 & & 41 & 18 & 41 & & 65 \\
\hline & 75 & 496 & 464 & 442 & & 38 & 56 & 6 & & 32 \\
\hline & 100 & 496 & 465 & 434 & 413 & 28 & 45 & 17 & 10 & 13 \\
\hline \multirow{4}{*}{$1: 1 \mathrm{~b}$} & 25 & 498 & & & & 100 & & & & \\
\hline & 50 & 501 & 485 & 469 & & 38 & 22 & 40 & & 69 \\
\hline & 75 & 496 & 464 & 442 & & 39 & 55 & 5 & & 35 \\
\hline & 100 & 497 & 465 & 435 & 414 & 28 & 44 & 18 & 10 & 14 \\
\hline \multirow{4}{*}{$3: 1 b$} & 25 & 497 & & & & 100 & & & & \\
\hline & 50 & 499 & 485 & 469 & & 39 & 22 & 39 & & 79 \\
\hline & 75 & 494 & 461 & 442 & & 41 & 52 & 7 & & 45 \\
\hline & 100 & 493 & 465 & 431 & & 29 & 36 & 35 & & 21 \\
\hline \multirow[t]{4}{*}{$7: 1$} & 25 & 496 & & & & 100 & & & & \\
\hline & 50 & 489 & 468 & & & 72 & 28 & & & 89 \\
\hline & 75 & 490 & 458 & 440 & & 44 & 52 & 4 & & 54 \\
\hline & 100 & 488 & 462 & 431 & & 33 & 30 & 37 & & 27 \\
\hline
\end{tabular}

${ }^{a}$ Data for uncoupled (free) FITC given for both low $[\mathrm{L}]$ and high $[\mathrm{H}]$ concentrations of label.

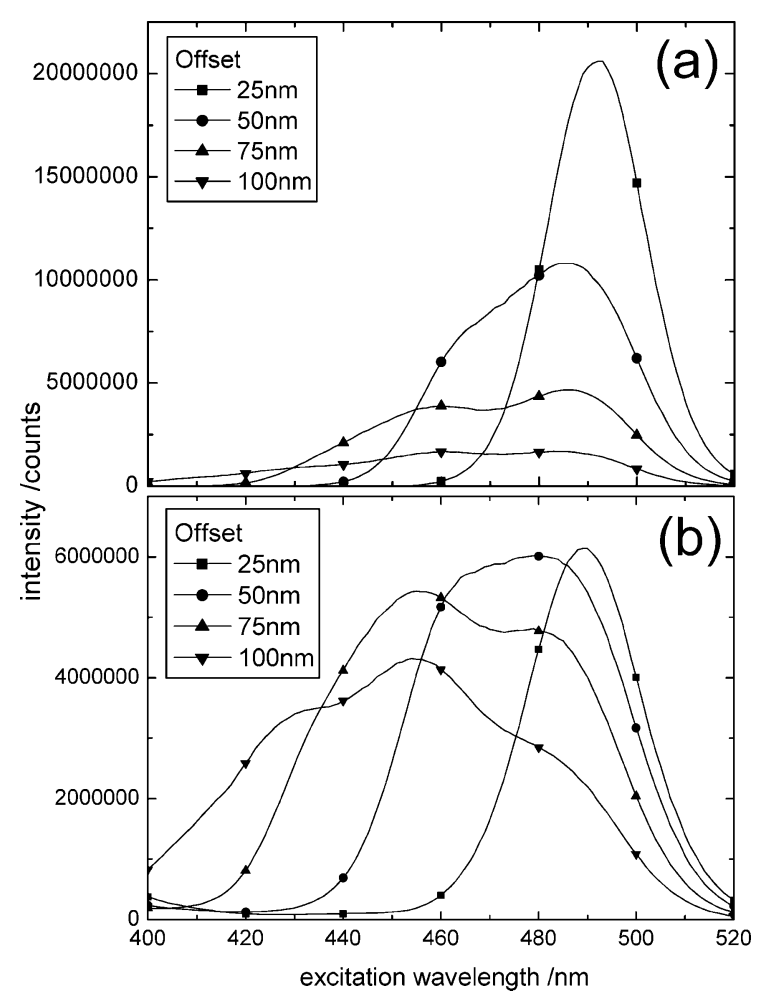

Fig. 4 Synchronous scan fluorescence spectra for (a) uncoupled FITC in PBS and (b) uncoupled FITC in PBS incorporated into AOT inverse micelles $\left(\omega_{0}=5\right)$, at different offsets between the excitation and emission wavelengths.
The spectra of FITC labelled BSA for representative labelling ratios are shown in Fig. 5 and the outcome of spectral decomposition is given in Table 2. The representative spectra in Fig. 5 show that an increasing labelling ratio has little effect on the form of the $25 \mathrm{~nm}$ offset spectra, and only a marginal reduction in the peak emission wavelength $(\sim 1 \mathrm{~nm})$ is seen. It is also worth noting that the width (at half maximum) of the curve fitted to the $25 \mathrm{~nm}$ offset spectra remained constant (20-21 nm) all throughout, which illustrates that the actual Stokes' shift is constant. ${ }^{23}$ This can imply that the apparent increase observed in Table 1 is caused by the effect of the growth of the shoulder, as also indicated by the decomposition of the absorption and fluorescence spectra. For longer wavelength separations, on increasing labelling ratio, there is a tendency towards the form of that obtained for a high concentration uncoupled FITC sample (Fig. 4b), although coupled and uncoupled FITC exhibit different wavelengths. The relative intensities of the longer wavelength offset spectra increase with labelling ratio. For example, at the lower ratio the $50 \mathrm{~nm}$ offset spectrum is $65 \%$ of that of the $25 \mathrm{~nm}$ offset spectrum, this increases to $89 \%$ for the $7: 1$, FITC: BSA, sample. Similar trends are seen for the two other offsets and this fact can relate to the increased intensity of the shoulder in both the absorption and fluorescence spectrum. When a wavelength separation of $50 \mathrm{~nm}$ is used its form changes and at the higher labelling ratio the decomposition only required the sum of two Gaussian spectra to obtain an acceptable fit, as seen for a high concentration of FITC. Thus, interactions between different FITC molecules can be observed and followed using this technique and again significant interactions appear beyond a labelling ratio of $c a .3: 1$. 


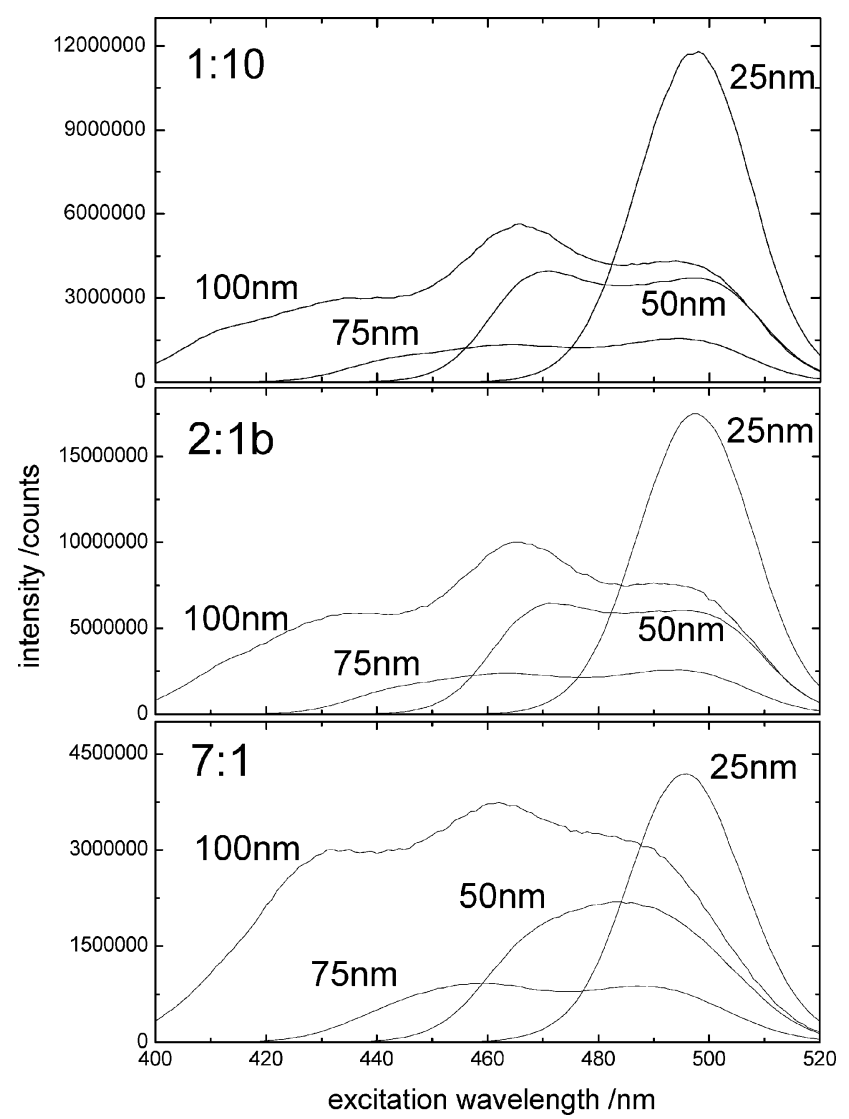

Fig. 5 SFS data from selected samples from the three regions (A, B, C) showing the spectra obtained at four different offsets. All the $100 \mathrm{~nm}$ offset spectra are shown multiplied by ten.

\section{Time-resolved fluorescence}

The fluorescence decay of coupled FITC is multi-exponential, see Table 3, and at lower labelling ratios requires the sum of two exponential components, one with a decay time around $4 \mathrm{~ns}$ and another just under a nanosecond, which accounts for roughly a third of the decay. The former is close to that obtained for the uncoupled FITC, where a value of 4.05 ns was recovered, in agreement with a previous measurement. ${ }^{24}$ This bi-exponential behaviour has previously been reported for FITC coupled to various proteins. ${ }^{25-28}$ The shortening of the lifetime has previously been attributed to quenching by certain amino acids (principally tryptophan). ${ }^{29}$ These recovered decay parameters remain more or less constant until a labelling ratio of $3: 1$ is reached, although it should be noted that there is a worsening in the goodness of fit, and beyond this ratio it is necessary to include a third component to obtain an acceptable fit to the decay. This additional decay component has also been observed for a FITC-HSA conjugate at a ratio of $7: 1 .{ }^{27}$ At this moment in time, for simplicity, it is useful to make use of an average lifetime and a fuller analysis of the decay kinetics is at present underway. The effect of the labelling ratio on the average lifetime can be seen in Fig. 6 and is strikingly similar to that obtained from the anisotropy data, which is supportive of an energy transfer process. These results plus the SFS data point to the fact that, although commencing around a labelling ratio close to $2: 1$ energy migration really becomes dominant beyond a ratio of $3: 1$.

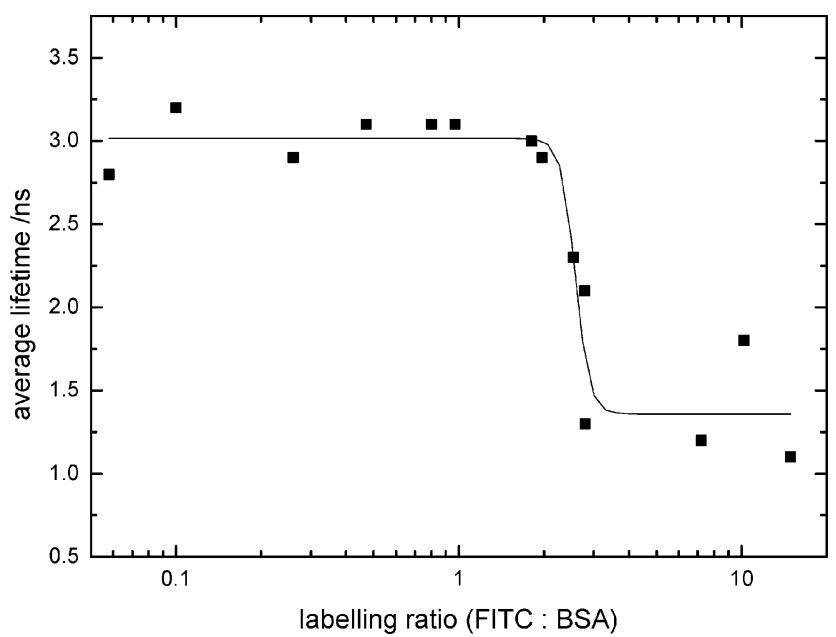

Fig. 6 Average decay time for FITC at different FITC: BSA labelling ratios. A sigmoidal fit has been added for guidance.

From the time-resolved data, if Förster type kinetics are assumed, it is possible to make an estimate for the average distance between FITC molecules on BSA. Fig. 7 shows the relationship between the average decay time and distance. However, if the lifetime for uncoupled FITC (4.05 ns) is used (solid line in Fig. 7),

Table 3 Time-resolved decay parameters for the decay of FITC attached to BSA at different ratios. The excitation was at $490 \mathrm{~nm}$ and the emission was monitored using a $550 \mathrm{~nm}$ cut off filter

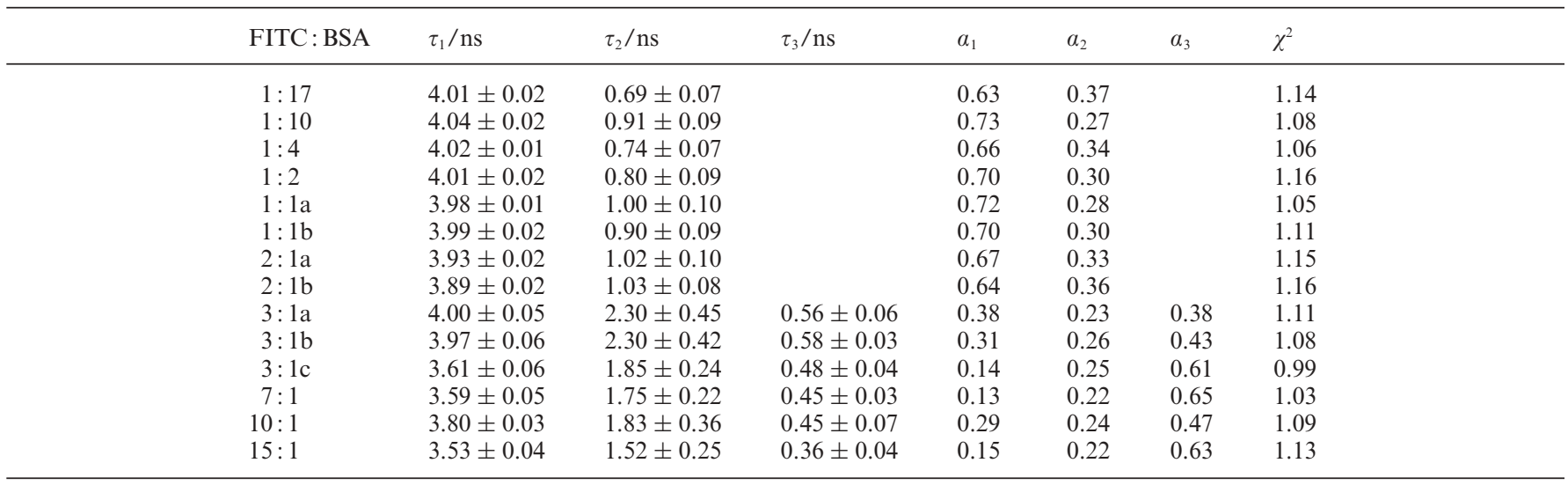




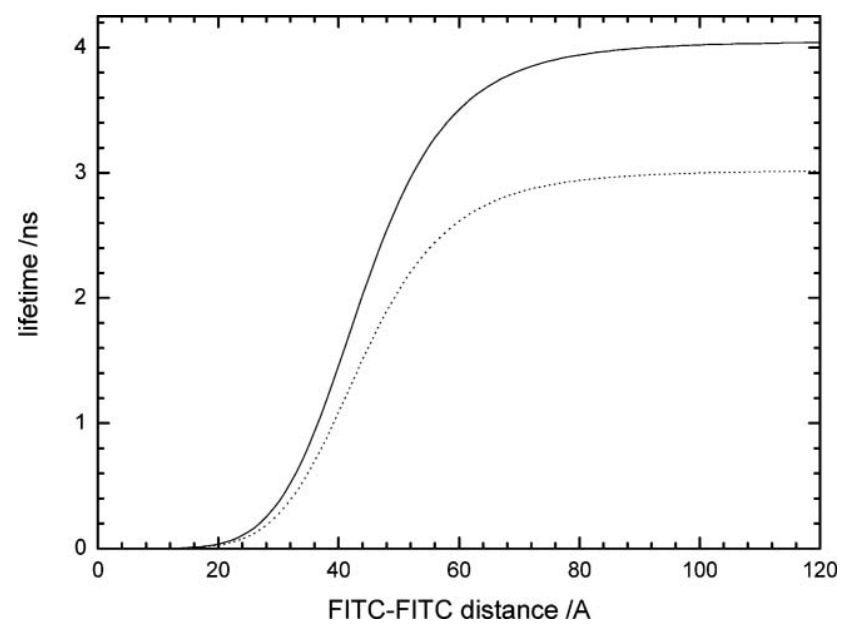

Fig. 7 Calculation of the decay time $(\tau)$ with the label-label distance $(R)$. The solid line assumes an unquenched decay time of $4.05 \mathrm{~ns}$, while the dotted line is for a decay time of $3.02 \mathrm{~ns}$. Lines calculated using $1 / \tau_{\text {calc }}=$ $(1 / \tau)+\left(1 / \tau\left(R_{0} / R\right)^{6} . R_{0}\right.$ is taken as $44 \AA$.

this implies that there is always some quenching (as the average decay time is nearly a nanosecond shorter) corresponding to a distance of $c a$. $50 \AA$. The possibility exists that, even at low labelling ratios, more than one FITC tag is attached to BSA and that this causes a reduction in fluorescence lifetime. However, a trend would then be expected in the pre-exponential components and the average lifetime of the under labelled samples. These data appear consistent and as there is the possibility that coupling of the dye to the protein produces a reduction in average lifetime it was decided to use the average decay time in the under labelled region as a reference to calculate the average distance between FITC tags (dotted line in Fig. 7). This means that the average distance between FITC molecules at the labelling ratio of $3: 1$ is ca. $53 \AA$ and that at the highest labelling ratio a distance of $40 \AA$ is found. These values appear sensible in relation to the size of the protein. However, this of course is a simplistic approach, albeit useful and readily applicable (a similar method has been used with silica colloids), ${ }^{19}$ and we are at present involved in further analysis of the time-resolved data.

\section{Addition of urea}

In order to perturb the system, the influence of urea $(\sim 6.5 \mathrm{M})$ was studied, as this is known to completely unfold serum albumin at concentrations higher than $6 \mathrm{M}$, where a high degree of random coil is formed. ${ }^{30}$ Samples representative of regions A, B and C (Fig. 3)were chosen, i.e. ratios FITC:BSA of $1: 4,1: 1$ and $3: 1$. On addition of urea the emission spectrum shape was found to broaden and shift to longer wavelengths, see Fig. 8, while the peak

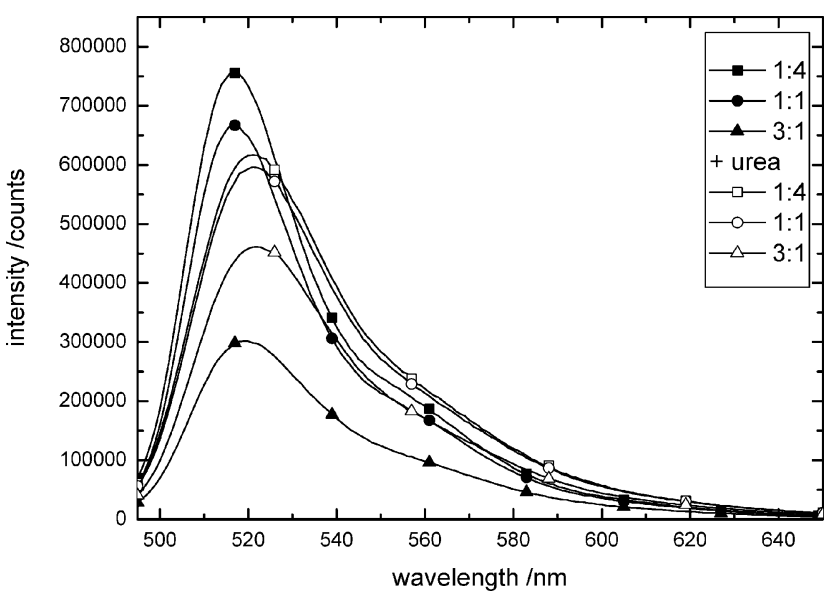

Fig. 8 Effect of the addition of $6.5 \mathrm{M}$ urea on the emission of FITC, labelled at different ratios to BSA. Solid symbols before addition, open symbols after addition of urea. Spectra have been corrected for differences in absorbance to give relative quantum yields.

absorption remained at $500 \mathrm{~nm}$ for each. A synchronous scan at an offset of $25 \mathrm{~nm}$ also yielded a single Gaussian curve of FWHM ca. $25 \mathrm{~nm}$, which is broader than that in the absence of urea and indicative in a change in the Stoke's shift. The decomposition recovered comparable results in all three cases, which were similar to those obtained for the more dilute uncoupled FITC sample.

Previous work on dilute fluorescein solutions has shown that urea has little effect on the dyes quantum yield. ${ }^{31}$ When we compare the yields (by integration of the spectra given in Fig. 8), using that of the region A, sample without urea for reference, as seen in Table 4, it is noticeable that the higher labelled sample has a much lower yield, with that of the $1: 1$ sample (marginally over labelled) slightly lower as would be expected because of the effect of energy migration. Also given in Table 4 are the values for the steady state anisotropy and the average lifetime. On addition of urea, although a change is observed in the spectrum shape, the yield for the under labelled sample is constant, while that of the $1: 1$ sample increases close to it, as would be expected as the protein unfolds and the distance between FITC molecules increases, with a concomitant reduction in energy migration. A major change is observed for the over labelled sample and its relative yield doubles. This tendency is also seen in the average lifetime and corresponds to an increase in the average separation of $c a .19 \AA$ (considering the dotted curve in Fig. 7) between the FITC tags.

The steady state anisotropy, which is also a measure of energy migration, exhibits the expected trend, but on addition of urea all the anisotropy values appear to converge. An explanation can be that as well as reflecting the effect of energy migration, as the protein structure unfolds the FITC is freer to rotate and this effect is now dominant in the observed anisotropy value. A time-resolved

Table 4 Comparison of FITC fluorescence properties on addition of $6.5 \mathrm{M}$ urea

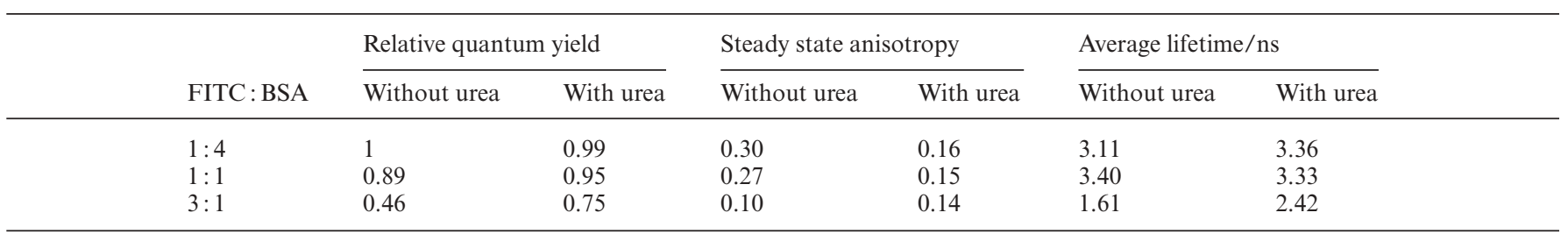


anisotropy measurement of the under labelled sample gave a rotational correlation time of $\sim 28 \mathrm{~ns}$ (standard deviation $4 \mathrm{~ns}$, initial anisotropy $0.31, \chi^{2}=1.07$ ), which we relate to the rotation of the entire protein. On the addition of urea the anisotropy decay required the sum of two exponentials to fit the data. The recovered rotational correlation times were $0.7 \mathrm{~ns}$ and $9.0 \mathrm{~ns}$, in roughly equal proportions (initial anisotropy $0.31, \chi^{2}=1.03$ ). This is in keeping that treatment with urea produces a very loose protein structure ${ }^{30}$ in which the FITC label is freer to rotate and even the protein may exhibit a faster rotational motion because of its unravelled form. The initial anisotropy, however appears unchanged and this shows that although the steady state anisotropy is useful, it is limited if rotation of the molecule comes into play. The lifetime, however, remains a good indication of the extent of the probe interaction and hence suitable for monitoring changes in conformation, with support from SFS.

\section{Conclusion}

The use of different fluorescence techniques have proved useful for the elucidation of the photophysics of the common protein label FITC, when attached to a protein in differing quantities. There is clear evidence of interaction between the probe molecules, particularly beyond a labelling ratio of $3: 1$ (FITC: BSA). This has been used as a tool to elucidate the average distances between the labels on a protein, allowing one to monitor changes in protein conformation.

\section{Acknowledgements}

Portuguese Foundation for Science and Technology (FCT) through funds from the POCTI and/or FEDER Programmes (Grant SFRH/BPD/17584/2004) and the European Union funded STREP project HIPPOCRATES (NMP3-CT-2004500283). This work was also carried out under the scope of the European NoE EXPERTISSUES (NMP3-CT-2004-500283).

\section{References}

1 J. R. Lakowicz, Principles of fluorescence spectroscopy, Plenum Press, New York, 1983.

2 B. Valeur, Molecular Fluorescence, Principles and Applications, WileyVCH, Weinheim, 2002.

3 V. Levi and F. L. G. Flecha, Reversible fast-dimerization of bovine serum albumin detected by fluorescence resonance energy transfer, Biochim. Biophys. Acta, 2002, 1599, 141-148.

4 J. Karolin, M. Fa, M. Wilczynska, T. Ny and L. B-Å. Johansson, Donor-donor energy migration for determining intramolecular distances in proteins: I. Application, of a model to the latent plasminogen activator inhibitor-1 (PAI-1), Biophys. J., 1998, 74, 11-21.

5 J. Karolin, P. Hägglöf, T. Ny and L. B.-Å. Johansson, Donor-donor energy migration (DDEM) for determining intramolecular distances in proteins. II. The, effect of partial labelling, J. Fluoresc., 1997, 7, 331-339.

6 S. Kalinin and L. B.-Å. Johansson, Utility and considerations of donordonor energy migration as a fluorescence method for exploring protein structure-function, J. Fluoresc., 2004, 14, 681-691.

7 S. Kalinin, J. G. Molotkovsky and L. B.-Å. Johansson, Distance measurements using partial donor-donor energy migration within pairs of fluorescent groups in lipid bilayers, J. Phys. Chem. B, 2003, 107, 3318-3324.

8 X. Duan, Z. Zhao, J. Ye, H. Ma, A. Xia, G. Yang and C.-C. Wang, Donor-donor energy migration measurements of dimeric DsbC labeled at its N-terminal amines with fluorescent probes: A study of protein unfolding, Angew. Chem., Int. Ed., 2004, 43, 4216-4219.

9 R. Sjöback, J. Nygren and M. Kubista, Absorption and fluorescence properties of fluorescein, Spectrochim. Acta, 1995, A51, L7-L21.

10 A. Kawski, Excitation energy transfer and its manifestation in isotropic media, Photochem. Photobiol., 1983, 38, 487-508.

11 J. N. Miller, Fluorescence energy transfer methods in bioanalysis, Analyst, 2005, 130, 265-270.

12 R. S. Knox, Theory of polarization quenching by excitation transfer, Physica, 1968, 39, 361-386.

13 D. J. S. Birch and R. E. Imhof, in Topics in fluorescence spectroscopy, vol. 1, techniques, ed. J. R. Lakowicz, Plenum Press, New York, 1991.

14 S. Rubio, A. Gomez-Hens and M. Valcarcel, Analytical applications of synchronous fluorescence spectroscopy, Talanta, 1986, 33, 633-640.

15 T. M. R. Viseu, G. Hungerford, A. F. Coelho and M. I. C. Ferreira, Dye-host interactions for local effects recognition in homogeneous and nanostructured media, J. Phys. Chem. B, 2003, 107, 13300-13312.

16 G. Hungerford, A. Rei and M. I. C. Ferreira, Studies on the interaction of nile red with horseradish peroxidase in solution, FEBS J., 2005, 272, 6161-6169.

17 M. M. Puchalski, M. J. Morra and R. von Wandruszka, Assessment of inner filter effect corrections in fluorimetry, Fresenius J. Anal. Chem., 1991, 340, 341-344.

18 E. W. Voss, Jr., J. C. Crony and D. M. Jameson, Discrete bathochromic shifts exhibited by fluorescein ligand bound to rabbit polyclonal antifluorescein fab fragments, J. Protein Chem., 2002, 21, 231-241.

19 A. Imhof, M. Megens, J. J. Engelberts, D. T. N. de Lang, R. Sprik and W. L. Voss, Spectroscopy of fluorescein (FITC) dyed colloidal silica spheres, J. Phys. Chem. B, 1999, 103, 1408-1415.

20 K. K. Rohatgi and G. S. Singal, Nature of bonding in dye aggregates, J. Phys. Chem., 1966, 70, 1695-1701.

21 I. López Arbeloa, Dimeric and trimeric states of the fluorescein dianion. Part 1.-Molecular structures, J. Chem. Soc., Faraday Trans. 2, 1981, 77, 1725-1733.

22 I. López Arbeloa, Dimeric and trimeric states of the fluorescein dianion. Part 2.-Effects on fluorescence characteristics, J. Chem. Soc., Faraday Trans. 2, 1981, 77, 1735-1742.

23 T. Vo-Dinh, Multicomponent analysis by synchronous luminescence spectrometry, Anal. Chem., 1978, 50, 396-401.

24 A. J. Abott, E. Amler and W. J. Ball, Jr., Immunochemical and spectroscopic characterization of two fluorescein 5 '-isothiocyanate labelling sites on $\mathrm{Na}^{+}, \mathrm{K}^{+}$-ATPase, Biochemistry, 1991, 30, 1692-1701.

25 E. Lewitzki, E. Schick, R. Hutterer, F. W. Scheider and E. Grell, Characterising protein conformation transitions of Na,K-ATPase with antibodies by fluorescence spectroscopy, J. Fluoresc., 1998, 8, 115-119.

26 C. Deka, B. E. Lehnert, N. M. Lehnert, G. M. Jones, L. A. Sklar and J. A. Steinkamp, Analysis of fluorescence lifetime and quenching of FITC-conjugated antibodies on cells by phase-sensitive flow cytometry, Cytometry, 1996, 25, 271-279.

27 J. R. Lakowicz, J. Malicka, S. D’Auria and I. Gryczynski, Release of the self-quenching of fluorescence near silver metallic surfaces, Anal. Biochem., 2003, 320, 13-20.

28 M. Heidecker, Y. Yan-Marriott and G. Marriott, Proximity relationships and structural dynamics of the phalloidin binding site of actin filaments in solution and on single actin filaments on heavy meromyosin, Biochemistry, 1995, 34, 11017-11025.

29 N. Marmé, J.-P. Knemeyer, M. Sauer and J. Wolfrum, Inter- and intramolecular fluorescence quenching of organic dyes by tryptophan, Bioconjugate Chem., 2003, 14, 1133-1139.

30 B. Farruggia and G. A. Picó, Thermodynamic features of the chemical and thermal denaturations of human serum albumin, Int. J. Biol. Macromol., 1999, 26, 317-323.

31 K. K. Rohatgi and G. S. Singal, The influence of urea on the fluorescence of aqueous dye solutions, J. Phys. Chem., 1963, 67, 2844 2846. 